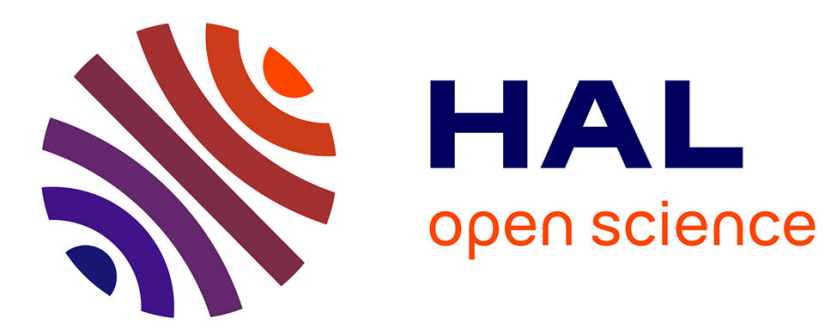

\title{
Territorialisations de l'islam dans l'espace d'organisation Sahel-Sahara-Méditerranée
}

\author{
Anne Ouallet
}

\section{To cite this version:}

Anne Ouallet. Territorialisations de l'islam dans l'espace d'organisation Sahel-Sahara-Méditerranée. L'Information géographique, 2019. hal-02358373

\section{HAL Id: hal-02358373}

\section{https://hal.science/hal-02358373}

Submitted on 11 Nov 2019

HAL is a multi-disciplinary open access archive for the deposit and dissemination of scientific research documents, whether they are published or not. The documents may come from teaching and research institutions in France or abroad, or from public or private research centers.
L'archive ouverte pluridisciplinaire HAL, est destinée au dépôt et à la diffusion de documents scientifiques de niveau recherche, publiés ou non, émanant des établissements d'enseignement et de recherche français ou étrangers, des laboratoires publics ou privés. 


\title{
Territorialisations de l'islam dans l'espace d'organisation Sahel-Sahara-Méditerranée
}

\author{
Anne Ouallet, Université Rennes 2, UMR ESO
}

L'Islam est un fait englobant dans l'espace Sahel-Sahara-Méditerranée. Il y est structurant pour les espaces et les sociétés. L'Islam, compris comme ensemble civilisationnel, participe à refaçonner les territoires en s'inscrivant dans le cadre de spatialités différenciées, particulièrement repérables dans des aires d'influences, des réseaux et des nodalités fonctionnant comme repères culturels, économiques, sociaux, politiques et se déployant à différentes échelles : de l’international au microlocal et inversement.

Les modes de territorialisation de l'islam, décliné comme religion, peuvent être concurrents ou complémentaires et s’inscrire dans des projets qui dépassent le spirituel par une instrumentalisation idéologique et politique.

Dans tous les cas, les spatialités sont à lire sur la longue durée et comme un palimpseste imprégné de séquences et d'inscriptions associées à des temporalités plus courtes liées à la multiplication des territoires du religieux, à leurs alliances ou à leurs mises en concurrence.

Dans l'espace Sahel-Sahara-Méditerranée, il s’agit finalement de spatialisations toujours en cours et dont les modes de territorialisations bouleversent les repères des États modernes en dépassant totalement leurs frontières et en les recomposant de l'intérieur.

\section{Une territorialisation sur la longue durée}

L’espace du Sahel à la Méditerranée en passant par le Sahara est totalement imprégné par l’Islam, d'une part parce que ce dernier y est une donnée majoritaire et d'autre part parce qu'il se déploie dans des sociétés déjà globalement religieuses et en cours de réislamisation. Arrivé au Maghreb dès sa première expansion, l'islam a très vite gagné l’Afrique subsaharienne. Il y était présent dès le début du VIII ${ }^{\text {ème }}$ siècle. Il y a été diffusé notamment par les troupes du général 'Uqba ben Nâfi', envoyé du calife omeyyade de Damas, Mu'awiya $\mathrm{I}^{\text {er }}$, et dont les arabes Kuntas de Gao et de Tombouctou (Nord du Mali) se disent actuellement les descendants. Les Ibadites ont également œuvré à sa diffusion depuis l'Algérie.

L’islam s'est fondu localement dans les territoires tout en y apportant des idées et des repères neufs. Les idées qu'il colportait ont été véhiculées au fur et à mesure par les marchands et pèlerins cheminant de relais en relais le long de voies d'échanges reliant, dans les deux sens, les espaces sahélo-sahariens à la Méditerranée et même bien au-delà. Les différents héritages font la richesse des territoires où il s'est durablement implanté.

\section{Des lieux de distinction et des aires d'influence façonnés par les héritages et les rencontres}

Les héritages sont bien sûr ceux des différentes périodes de l’islam, mais ils puisent aussi toujours aux sources des périodes préislamiques. Partout, il existe un substrat de religions locales. La richesse des expressions de l'Islam est particulièrement observable lors des cérémonies, pèlerinages, visites saintes qui canalisent nombre de croyants en certains lieux distingués par leur sainteté. Ces héritages sont aussi ceux des écoles juridiques de l'islam dont l'influence spatiale est davantage lisible, cette fois-ci, en termes d'aires d'influence qui parfois s'entremêlent.

De même que La Mecque préislamique a laissé ses empreintes dans La Mecque musulmane (focalisation sur la pierre noire de la Kaaba par exemple), chaque lieu du Sahel, du Sahara ou des espaces de l'Afrique méditerranéenne est un condensé des mémoires locales qui, depuis l'apparition de l'islam, l'ont accompagné dans ses interprétations et expressions. Nombreux sont les exemples localisés de tels héritages. Certains ont acquis plus de renommée que d'autres en capitalisant des savoirs liés à l'islam. C'est ainsi qu'à Djenné, au centre du Mali, la ville entière porte haut la réputation maraboutique dont elle use comme d'une ressource dans l'espace ouest africain (Ouallet, 
2013). Les marabouts ${ }^{1}$ de la ville ont une réputation qui dépasse les frontières du Mali. Quant aux maçons de Djenné, ils ont exporté leur savoir dans tout l'espace ouest sahélien. Appartenant à une caste crainte et respectée, ils puisent leurs incantations magiques à la fois des connaissances qu'ils retirent de l'islam tel qu'approprié dans cette éminente cité maraboutique et dans la période préislamique. Au Maroc, la petite ville de Moulaye Idriss ${ }^{2}$, devenue cité sainte et principal site de pèlerinage du pays abrite le mausolée du fondateur de la dynastie Idrisside. Beaucoup s’y rendent en visites pieuses et dans des buts thérapeutiques, probablement héritage des pouvoirs attribués au dieu Frugifer de l'Antiquité. En Éthiopie, dans la province du Balé au Sud de la ville de Harar, a lieu l'un des plus grands pèlerinages musulmans de la Corne de l'Afrique : celui de Cheikh Hussein. Les pèlerins se rendent sur ce qui est déclaré être le tombeau d'Abdel Qader El Jilani originaire de Jilan (Nord de l'Iran actuel) et saint fondateur de la confrérie qadriyya ${ }^{3}$. Les rituels observés localement incluent des prosternations devant le feu. Trois exemples parmi une multitudes d'autres. Tous ces lieux attirent, parfois de façon éphémère, des foules séduites par leur distinction assurée sur le plan religieux, mais aussi par diverses autres opportunités, telles que retrouvailles, possibilités d'échanges, de commerce, de guérison, de se construire une notoriété, etc. Ils forment des repères essentiels, chacun à leur niveau de notoriété, et sont un des fondements de l'animation des territoires.

A une échelle spatiale plus vaste observée sur l'ensemble de l'aire étudiée et jusqu'au Proche-Orient, les pratiques sont influencées par les quatre principales écoles juridiques de l’islam sunnite. Ces dernières véhiculent des règles qui octroient une plus ou moins grande liberté aux jurisconsultes et encadrent les rites. Finalement au-delà des codes juridiques, les pratiques associées permettent d'identifier des espaces d'influence. L'école hanafite héritée de l'empire ottoman est réputée être la plus libérale. Elle est désormais minoritaire, subsiste dans d'anciennes dépendances de l'Empire ottoman, notamment en Algérie et en Tunisie, mais y a été plutôt supplantée par l'école malékite, plus conservatrice, mais très consensuelle, influencée par l'islam pacifique de la première période médinoise pendant laquelle l'islam était en position d'arbitre entre les juifs, les chrétiens et les musulmans ${ }^{4}$. Le malékisme est très implanté au Maroc, en Afrique occidentale (Sénégal, Mali, Niger) et jusqu'au Soudan. La politique actuelle du Royaume chérifien marocain s’appuie sur ce malékisme pour développer son influence au Sud du Sahara, notamment pour essayer de contrebalancer le radicalisme intransigeant d'un hanbalisme ${ }^{5}$ conquérant, référence du wahhabisme saoudien qui déferle depuis quelques décennies sur l'Afrique et, plus récemment, du salafisme qatari. Le malékisme a majoritairement favorisé des interprétations pacifiques et a imprégné les confréries. Cependant, l'influence de jurisconsultes hanbalites a introduit depuis plusieurs siècles déjà des compréhensions exclusives et intolérantes de l'islam au Maghreb, au Sahara et jusqu'au cœur du Sahel. Ces influences sont réexploitées par l'offensive actuelle de l'islam radical.

La quatrième école, le chaféisme, est, quant à elle, davantage développée vers les pays du Golfe et le pays de Cham (Syrie, Palestine).

Les territoires sont partout socialement et politiquement construits. Leurs dimensions conceptuelles, matérielles et vécues sont à lire à l'aune des héritages locaux, des apports extérieurs et des capacités d'encadrement. Du Sahel à la Méditerranée, ils ont été consolidés dans le cadre d'organisations socio-politiques qui doivent leur construction au contrôle des circuits à longue distance. C'est leur adhésion à l'islam qui leur a permis d'assurer ce contrôle sur les voies de circulation et d'en tirer

\footnotetext{
${ }^{1}$ Religieux de l'islam, à qui sont souvent reconnus des pouvoirs particuliers.

${ }^{2}$ A proximité de Meknès.

${ }^{3}$ Décrite ci-après dans l'article

${ }^{4}$ On peut distinguer une première période mecquoise où l'islam minoritaire coexistait pacifiquement avec les religions préexistantes dans une Mecque globalement encore polythéiste. Succède une première période médinoise où l'islam a pris de l'influence et est régulièrement sollicité en arbitrage entre les religions monothéistes (Muhammad est à cette époque à Médine). L'islam devient ensuite majoritaire et entre dans une phase d'expansion plus belliqueuse, à Médine comme à La Mecque et justifie ses conquêtes territoriales par le djihad.

${ }^{5}$ Le rite hanbalite a été diffusé en Arabie Saoudite depuis Muhammad Ibn ‘Abd al-Wahhab (1703-1792).
} 
leur richesse.

\section{Des réseaux et des formations socio-spatiales dominés par l'islam}

L'espace Sahel-Sahara-Méditerranée est, dès les premiers siècles de l'islam, intégré dans ou relié à un ensemble plus vaste qui, à partir de la péninsule arabique connecte des territoires depuis la Chine jusqu'au Maghreb, " couchant », et au Sahel, "rivage " sud du Sahara, grâce notamment aux réseaux des voies de commerce balisées par des villes relais qui opèrent comme des charnières (Retaillé, 2018).

En Afrique, l'expansion de l'islam a très tôt emprunté les itinéraires transsahariens parcourus précédemment, en les réajustant dès que nécessaire. Côté Sahel, la vallée du Sénégal et la boucle du fleuve Niger ont également été des axes privilégiés de propagation de l'islam. Les voies de circulation préexistantes ont été confortées, au besoin réadaptées, en épousant les contextes les plus favorables. Du VIII ${ }^{\text {ème }}$ au XVI ${ }^{\text {ème }}$ siècles, on observe ainsi une translation de l'Ouest vers l'Est des « empires de la route » (Retaillé, 2018). La prise de contrôle des routes transsahariennes anticipe le déplacement spatial d'Ouest en Est des cœurs économiques et politiques des empires du Ghana (750-1204), du Mali (1235-1546) et du Songhoï (1464-1591). Plus à l’Est, les royaumes du Haoussa, du Sokoto, du Bornou, du Ouaddaï et du Darfour se sont développés aussi grâce aux réseaux de l'islam.

Certains réseaux et nœuds préexistaient à l’islam : la cité bimillénaire de Djenné au Sahel, Le Touat pour le Sahara, Ouarzazate et Biskra pour le Maghreb ou encore Héliopolis et Giza intégrés actuellement dans la mégapole du Caire pour l'Égypte. Ils ont été confortés par son enracinement. D’autres villes, comme Tombouctou, Agadès, Kairouan ou encore Al Qaïra (quartier Fatimide du Caire) sont constitutifs du développement de pouvoirs directement liés à l'islam.

\section{Routes de commerce et trafics}

Les voies méridiennes (Retaillé, 2013, carte 7) sont sur la longue durée les plus parcourues car elles jouent sur la complémentarité des produits entre les puissantes organisations politiques de l'Afrique méditerranéo-saharienne (Fatimides, Mérinides, Saadiens) et celles des espaces sahélo-sahariens (du Ghana au Darfour). Durant l'âge d'or de ces empires, parmi les très diverses marchandises échangées se trouvaient de nombreux esclaves. La plupart des marchandises transportées ont évolué au fil des siècles et des contextes ainsi que les itinéraires. Par exemple, à la suite de la prise de la mine de sel de Teghazza par les saadiens en 1589, les voies joignant le Touat à la boucle du Niger par les salines ont été doublées d'un itinéraire reliant cette halte du centre du Sahara à Tadmekka et à Gao (Scheele, 2012) par la vallée du Tilemsi. Cette vallée du Tilemsi est actuellement une voie intense de trafics illégaux et est couramment empruntée pour rejoindre l'Adrar des Ifoghas au Nord Mali, véritable base des groupes djihadistes. La situation actuelle favorise la reprise du trafic d'êtres humains, migrants, esclaves. Il a été récemment dénoncé par exemple en Libye mettant en exergue l'exploitation de la vulnérabilité des migrants franchissant le Sahara ou voulant traverser la Méditerranée.

Jalonnant les rivages du désert, les villes relais de ces relations méridiennes sont également sur la longue durée des étapes incontournables sur les routes du pèlerinage à La Mecque empruntant alors aussi des trajectoires zonales. Les cheminements sahéliens permettaient de longer le désert d'Ouest en Est depuis Chinguetti jusqu'à la traversée de la Mer Rouge par Oualata, Tombouctou, Agadez, Abéché, Khartoum. Côté septentrional du désert, Marrakech, Fès, Ghardaïa, Kairouan, Sebkha, Le Caire, étaient quelques-uns des arrêts les plus valorisés par les pèlerins. Les pèlerinages vers La Mecque se font maintenant en avion et les anciennes routes de La Mecque sont plutôt réinvesties par des trafiquants.

Les voies de circulation sont devenues les routes de tous les trafics, dont la majorité sont illicites. Depuis l'instauration des États modernes, les contrebandiers ont toujours su jouer des frontières pour éviter les contrôles, se soustraire aux taxes, profiter des différentiels de prix. Du début des indépendances aux années 1980, la fraude se faisait surtout sur des produits manufacturés, du sucre, le lait en poudre « Lahda » et des cigarettes. Ils ont peu à peu fait place aux armes, à la drogue, aux 
migrants clandestins et au trafic de passeports et d'otages. Figure de ces trafiquants, Mokhtar Belmokhtar, surnommé " Monsieur Marlboro » ou encore le «Ben Laden du Sahara », a par exemple très vite élargi sa gamme de produits jusqu’à détenir des otages. Business dans lesquels trempent tous les djihadistes les plus en vue. Les otages enlevés au Niger, au Burkina Faso ou au Mali ont le plus souvent été séquestrés dans le bastion montagneux de l'Adrar des Ifoghas. Entre 2003 et 2016, environ 70 otages ont été enlevés dans l'espace sahélien et monnayés par Al Qaïda au Maghreb Islamique (AQMI). Entre 2007 et 2009, AQMI aurait perçu au moins douze millions d'euros (Fofana, 2017). Une source de l'ONU évalue le gain d'AQMI à près de 105 millions de dollars entre 2010 à 2013.

Les armes et la drogue sont un marché encore plus florissant. La cocaïne aurait ainsi rapporté à AQMI 15 milliards de dollars entre 2001 et 2014 et 900 millions d'Euros à Boko Haram (Fofana, 2017). La cocaïne emprunte ce qui est couramment appelé «l'autoroute 10 » qui part de Guinée Bissau où sont débarquées les cargaisons, puis longe le Sahel par le Sénégal, la Mauritanie, le Mali, le Niger, et remonte par le Sahara vers l'Algérie et la Libye. La réorientation des routes se fait en fonction de leur sécurisation par les passeurs et de l'adaptation aux circuits suggérés par des rentes qui sont connectées à des réseaux mondiaux illégaux. "Les narcotrafiquants sud-américains ont transformé l'Afrique de l'ouest en plaque tournante pour la distribution de cocaïne vers l'Europe, empruntant à rebours les voies du commerce triangulaire. Ils contribuent, avec les flux de migrants clandestins et la course aux ressources minières sahariennes, à un retournement spatial : le Sahara marginalisé par l'économie de traite atlantique retrouve une centralité, quitte à ce qu'elle soit largement clandestine ou criminelle » (Magrin, 2010). 30\% de la cocaïne consommée en Europe serait passée par AQMI (Fofana, 2017) et par l'espace du Sahel et du Sahara à la Méditerranée.

Les rentes liées au passage des trafics imposent un contrôle sur un espace débarrassé de ses constructions gênantes: profondeurs mémorielles, territoires vécus, donc appropriés par les populations, frontières des États modernes. Se revendiquant d'un islam hanbalite, Al Qaïda, au nom d'un retour aux sources de l'islam, prétend gommer tous ces héritages, dénoncés par l'association djihadiste comme étant des déviances. Les différents groupes djihadistes partagent cette même vision d'une re-territorialisation formatée selon leurs propres principes. Or, les héritages sont partout nombreux, marqués directement dans les territoires par des balisages, souvent des villes, des édifices, mais aussi des lieux dans lesquels les pratiques se sont enracinées, même quand elles sont éphémères.

Dans cet espace Sahel-Sahara-Méditerranée où la civilisation de l'Islam marque les territoires, les conflits sont à l'heure actuelle violents. Les fractures se construisent essentiellement entre une conception de l'islam portée par le hanbalisme et une autre où les rites malékites s'appuient sur les mémoires locales et les revivifient dès qu'ils le peuvent. L'islam confrérique $a$, dans la durée, un rôle considérable d'accompagnement dans les inscriptions territoriales. En Afrique, la territorialisation de l'islam sur sa temporalité la plus longue est essentiellement liée à un mode de diffusion qui s'appuie sur le soufisme.

\section{Enracinement et redéploiement de l'islam soufi dans les territoires}

Les voies soufies ont été largement soutenues et continuent à être investies par les confréries. La première confrérie à avoir été rapidement diffusée en Afrique est la Qadriyya, voie mystique encore majoritaire dans la Corne de l'Afrique. Elle a ensuite été largement supplantée par d'autres confréries qui se sont enracinées dans l’espace Sahel-Sahara-Méditerranée et s’y sont déployées.

\section{Déploiement confrérique}

Les réseaux confrériques ont appuyé l'expansion de la religion musulmane. Ils se sont, depuis, diversifiés et sont très actifs, notamment grâce aux zawiyya, lieux d'enseignement et de rencontre des adeptes. Actuellement, ils participent fortement au mouvement général de réislamisation. Les 
écoles de pensée et les rites véhiculés par ces réseaux ont été et continuent à être très efficaces dans la transmission de la mémoire et de la portée universaliste de l'islam sans obliger pour cela les musulmans à y adhérer par une déclaration individuelle. En Afrique, de nombreux musulmans n'appartiennent donc à aucune confrérie. Le système confrérique est actuellement beaucoup plus ancré au Sénégal que dans la plupart des autres zones sahélo-sahariennes et méditerranéennes.

Le plus ancien réseau confrérique est celui de la Qadriyya, voie mystique diffusée depuis Bagdad par Abdel Qader Al Jilani (1077-1166), soufi ayant grandi dans la capitale abbasside. Il a permis la diffusion de l'islam vers l'Afrique. La Qadriyya s'est très tôt fortement implantée dans la Corne de l'Afrique où elle reste la référence, a été diffusée en Afrique du Nord d'où elle a été répandue dans la zone saharo-sahélienne à partir de l'Algérie. Elle y reste actuellement très présente, notamment de la Mauritanie jusqu'au Nord du Nigeria, s’adaptant à chaque fois au milieu local. Ses adeptes se replacent par rapport à une chaîne de transmission, silsila, qui s’avère, pour eux, essentielle et les rattache au fondateur Abdel Qader Al Jilani. Au Balé, dans le bastion montagneux Sud de l'Éthiopie, c'est la mémoire de ce soufi qui rassemble les pèlerins de Cheikh Hussein. La croyance locale y a même replacé son tombeau ${ }^{6}$. La Qadriyya est aussi bien implantée dans les espaces héritiers de l'empire du Sokoto (Nord de l'actuel Nigeria) dirigé par le chef qadri Ousmane dan Fodio (1754-1817) et dont l'influence s'étend à présent jusqu'au Niger et au Mali Nord-oriental dans la région de Gao. Toujours au Mali, mais au centre cette fois-ci, dans le Delta intérieur du Niger, ce sont surtout les descendants des adeptes de Cheikou Amadou, fondateur de l'empire peul du Macina, qui se réfèrent à cette voie. Dans beaucoup d'endroits, les adeptes sont discrets, en opposition totale aux fidèles mourides du Sénégal. La confrérie mouride, mouvement de rénovation de la Qadriyya, est d'essence purement sénégalaise, mais a conquis le monde (Bava Sophie, 2014). Les talibés, qui vouent une admiration sans borne à leur cheikh fondateur Amadou Bamba et une obéissance totale à leur marabout, sont présents aussi bien aux États-Unis qu'en Europe ou encore en Asie. La réussite internationale des mourides s'extériorise partout au Sénégal et particulièrement dans leur capitale Touba, ville millionnaire qui jouit d'un statut d'extraterritorialité religieuse (Gueye, 2002). Chaque année y est organisé le magal commémorant le départ en exil de Cheikh Amadou Bamba. Il rassemble des millions de visiteurs, adeptes mourides bien sûr, mais aussi de nombreux fidèles d'autres confréries ou sans appartenance confrérique.

Plus récente, la Tijaniyya est issue du Maroc. Elle a été diffusée dans toute l'Afrique de l'Ouest aux $X I^{\text {ème }}$ et $X^{\text {ème }}$ siècles (Robinson, Triaud, 2000). La confrérie tijane s'est à cette époque imposée aux côtés de la qadriyya et l'a rapidement supplantée permettant de meilleures opportunités de branchements sur les réseaux renouvelés de l'islam. La période du djihad d'El-Hadj Oumar Tall ${ }^{7}$ en a permis une extension particulièrement rapide depuis le Sénégal jusqu'à Bandiagara à l'Est du Mali actuel. Ce conquérant a été l'un des opposants les plus farouches à la colonisation française, mais ses successeurs ont souvent su composer avec l'administration coloniale. La force de conciliation de ces marabouts a favorisé l'expansion rapide de leur confrérie. Les réseaux de la Tijaniyya ont alors prospéré pacifiquement tout au long du $\mathrm{XX}^{\text {ème }}$ siècle. Ils sont actuellement vigoureux du Maroc jusqu'au Niger et même au-delà (carte 1).

Fès au Maroc est le pôle spirituel et spatial de la confrérie. C'est là que se trouvent la zawiyya et le mausolée du père fondateur Cheikh Ahmed Tijane ${ }^{8}$ vers lequel convergent bien des pèlerins. La confrérie a ensuite été diffusée en dehors du Maghreb par de grands marabouts et leurs adeptes qui ont œuvré à sa propagation dans toute l'Afrique de l'Ouest. On peut ainsi suivre la Tijaniyya oumarienne en suivant les traces d'El Hadj Oumar Tall depuis le Fouta Tooro sénégalais (moyenne vallée du Sénégal où il est né) jusqu'à Bandiagara, ville près de laquelle la légende le fait disparaître dans une grotte ${ }^{9}$. La confrérie s'est ensuite progressivement scindée en plusieurs branches, chacune d'entre elles suivant un cheikh.

\footnotetext{
${ }^{6}$ Le mausolée d’Abdel Qader Al Jilani est plus habituellement situé à Bagdad.

${ }^{7}$ Djihad lancé en 1854.

${ }^{8}$ mort à Fès en 1815

${ }^{9}$ le 12 février 1864 à Deguimbéré
} 
Malick $\mathrm{Sy}^{10}$, réputé pour son érudition, en a été un grand diffuseur pacifique, de même qu'Abdoulaye Niasse, fondateur d'une branche qui se distingue rapidement par son envergure internationale. C'est un de ses fils, Ibrahim Niasse, qui a donné l'élan internationaliste de ceux que l'on nomme nyassènes en référence au nom de leur cheikh. Troisième branche distincte à l'intérieur de la Tijaniyya, la Hamawiyya rassemble les disciples de Cheikh Chérif Hamallah ${ }^{11}$, considéré par la France comme opposant et déporté plusieurs fois. Les adeptes de Hamallah ont essaimé un peu partout au Sahel, comme le décrit Amadou Hampaté Bâ (Ouallet 2018), grand témoin de l'époque et fervent partisan hamalliste.

Au bout du compte, la Tijaniyya sous ses différentes formes occupe un large espace ouest africain compris comme s'étendant du Maghreb jusqu'en Afrique soudano-sahélienne du Nord au Sud et du Sénégal au Nil d'Ouest en Est. Au Nord-Est du Mali elle est concurrencée par la Senussiyya particulièrement active dans les espaces allant du Niger et du Tchad jusque vers la Libye. Plus à l'Est encore, au Soudan, les mahdistes ont une aire d'influence significative. De nombreuses autres confréries encore viennent proposer des offres religieuses.

Chacune de ces confréries ou branches confrériques a favorisé la mise en place de réseaux de communication, de circulation des idées, des biens et des hommes. Au-delà, elles permettent le branchement sur des réseaux qui sont des réseaux socio-économiques, parfois branchés avec le politique.

Dans une période d'effacement de l'État et de concurrence exacerbée pour le contrôle des territoires et de leurs ressources, les confréries développent leur propre stratégie de marquage.

\section{Revivification des territoires par les confréries : exemple de la Tijaniyya}

L'observation des espaces de revivification de la Tijaniyya apporte des éléments intéressants sur les territorialisations à l'œuvre actuellement. Certains réseaux ponctués de lieux déjà considérés comme saints sont réactivés. D'autres lieux sont nouvellement distingués par des entrepreneurs religieux qui y organisent des célébrations, telles que les ziyara qui sont des visites sur les tombeaux. Ils viennent s'insérer dans de nouvelles logiques de regroupements et de circulation. Le long des réseaux cheminent pèlerins et commerçants, les deux catégories étant en général associées. Fès continue à s'imposer comme pôle de premier plan (carte 2). L'intensification des flux entre le Sahel et la ville marocaine a été repérée par les acteurs économiques. Les agences de voyages sénégalaises mettent en avant des circuits intégrant quête spirituelle sur les chemins de la Tijaniyya avec pour point d'orgue Fès et opportunités d'activités économiques. Certains itinéraires proposent un passage par la zawiyya de Rabat Salé et par Casablanca. En 2008, Royal Air Maroc, la société nationale d'aviation du Maroc, a lancé un nouveau produit appelé Ziyâra Tijania visant à capter la clientèle malienne qui se rend à Fès. Un peu partout, les stratégies mémorielles développées s'organisent autour de mobilisations festives et religieuses, permettent les échanges sociaux et économiques et au-delà servent d'ancrages territoriaux, voire de tribune politique.

Le Fouta Tooro, centré sur la vallée du fleuve Sénégal, foyer historique majeur de diffusion de la Tijaniyya, montre parfaitement ce phénomène, observable aussi dans beaucoup d'autres endroits. Alwar et Gaya, villages de naissance respectivement d'Oumar Tall et de Malick Sy ont été récemment revivifiés à travers des commémorations valorisant la mémoire des Cheikhs. Dans cet espace transfrontalier entre la Mauritanie et le Sénégal, les petites villes relais de l'islam sont tantôt côté mauritanien comme Boghé, tantôt côté sénégalais comme Matam, faisant fi des frontières étatiques actuelles qui apparaissent finalement comme surimposées. Chaque branche trouve ses propres repères (carte 2).

L'inscription spatiale des territoires oumariens suit fidèlement les traces du conquérant toucouleur depuis le Sénégal jusqu'à Deguimbéré, lieu de disparition mythique d'El Hadj Oumar situé à une dizaine de kilomètres de la ville de Bandiagara (centre Est du Mali actuel). Les villes qu'il a conquises, telles que Nioro du Sahel, Ségou ou encore Bandiagara restent des étapes emblématiques

\footnotetext{
$101855-1922$

${ }^{11} 1883-1943$
} 
de cette épopée et valorisées en tant que telle. Les maouloud ${ }^{12}$ et ziyara se multiplient un peu partout là où il y a possibilité de marquage mémoriel.

L'inscription spatiale de la Hamawiyya fait de Nioro du Sahel, localité où l'élu, Cheikh Chérif Hamallah, avait été révélé, son point focal. La déportation et les internements des hamallistes pendant la période coloniale en créant un essaimage a, a contrario de ce qui était recherché à l'époque, participé à la réactivation de réseaux transnationaux de la Hamawiyya. Kaedi en Mauritanie comme Gagnoa en Côte d'Ivoire connaissent une recrudescence particulière des activités religieuses de la branche.

La Tijaniyya par la descendance d'El Hadj Malick Sy a, elle, conforté Tivaouane, ancienne capitale du Royaume du Cayor, comme capitale des Tijanes. Un certain nombre de haut-lieux, tels que Mpal, Fass et Pire y sont directement rattachés. Cette dernière localité a demandé en 2017 à obtenir la reconnaissance du statut de «cité religieuse » qui lui permettrait alors d'accéder aux financements dédiés prévus par l'État sénégalais dans le cadre de son Programme de modernisation des cités religieuses. Ce programme appuie finalement un phénomène déjà très visible de reterritorialisation par le religieux.

La territorialité niassène est, elle, tout à fait spécifique. Elle reflète l'engagement internationaliste de cette branche confrérique. Les liens tissés entre Ibrahim Niasse, fils du Cheikh fondateur, et l'Émir du Sokoto, ont fait de Kano (actuel Nigeria) un centre important de diffusion niassène au Nigeria même et plus à l'Est. Les réseaux niassènes sont actuellement présents dans tout le Sahel jusqu'au Nil soudanais et sont étendus plus au Sud jusqu'en zone soudano-sahélienne. L'actuel Khalife niassène est souvent convié à jouer les intermédiaires dans les conflits, notamment au Nigeria où les attaques de Boko Haram sèment le chaos. En retour, les délégations de Nigerians se déplacent au siège de la branche confrérique, à Kaolack (Sénégal).

Les courants confrériques se sont en général inscrits et continuent à s'inscrire dans des déploiements pacifiques, mis à part les djihad du $19^{\text {ème }}$ siècle (Oumar Tall, Cheikou Amadou, Ousman Dan Fodio) qui jusqu'à maintenant sont réutilisés dans un sens plus ou moins belliqueux. Les prédicateurs qui se situent d'eux-mêmes hors confrérie sont, par contre, à l'évidence influencés avant tout par un islam qui puise dans le hanbalisme pour prescrire des normes autoritaires et revendiquent de nouvelles formes de gestion des territoires.

\section{Marquages et territorialisations de l'islam hanbalite}

Le malékisme est actuellement très fortement concurrencé par les wahhabites et les salafistes qui diffusent des normes radicales par le biais d'actions prosélytes, mais aussi en s'appuyant sur un djihad violent dont le but est de contrôler populations et territoires en se substituant à l'État. Katiba et khalifats sont les espaces référents des territorialisations djihadistes.

\section{Conquête des territoires par l’islam hanbalite}

Dans l'espace Sahel-Sahara-Méditerranée, les associations musulmanes ont été démultipliées. L'exemple de la progression de ces associations au Mali peut mieux faire comprendre la rapidité avec laquelle le pays a basculé dans un encadrement par un islam de plus en plus omniprésent dans la sphère publique.

La sphère religieuse a été boostée par toute une série d'offres nouvelles et elle a été largement politisée. Certaines associations religieuses ont connu un grand succès grâce au charisme exceptionnel de leur dirigeant, mais aussi parce que l'attente de propositions est grande, notamment parmi les très nombreux laissés pour compte du «développement ». Si certains leaders soufis restent clairement dans un islam qui plonge ses valeurs dans les racines locales, d'autres reprennent des arguments plus proches des salafistes, sans forcément s'en réclamer. Il en est ainsi de l'association Ançar Dine International de Chérif Ousmane Madani Haïdara. Cette association a

\footnotetext{
${ }^{12}$ Fête musulmane la plus célébrée dans l'espace du Sahel, du Sahara à la Méditerranée et contestée par les salafistes. Elle correspond à la fête célébrant la naissance du Prophète.
} 
gagné en popularité depuis une vingtaine d'années ${ }^{13}$ particulièrement chez les cadets sociaux, principalement les femmes et les jeunes, en dénonçant les vices de la société. Madani Haïdara développe un rigorisme qui puise dans le hanbalisme, mais garde un certain nombre de points de repères chez les confrériques, comme celui de la célébration de la fête de maouloud qui est la plus populaire. La stratégie de contrôle spatial d'Ançar Dine International est calquée sur le mode du maillage administratif de l'État. Son organisation en sièges, sections, sous-sections et comités s'intègre dans la hiérarchie d'un maillage spatial qui permet une organisation remontante vers le siège central situé à Bamako. Ce siège de Bamako centralise les statistiques et les rapports qui sont récupérés à partir des bureaux des comités locaux, puis transmis par les sous-sections et sections. La situation financière dont les éléments remontent également du local est contrôlée à Bamako. Ce siège décerne aussi des diplômes. Spatialement, l'association avait réussi à couvrir tout le Sud et le centre du Mali jusqu’à Sévaré et espérait conquérir le Nord, tout spécialement Tombouctou, quand elle a été arrêtée par la vague djihadiste. Ses adeptes investissent préférentiellement les espaces publics : parvis des mosquées, stades, sillonnent les rues avec des haut-parleurs.

Tout autre est le mode d'organisation de la Da'wa. D'origine pakistanaise, la Da'wa œuvre depuis une trentaine d'années à l'échelle micro-locale, au niveau de chaque individu et au niveau de l'insertion de l'individu dans les sociabilités de base, appelées "grins » au Mali : regroupements d'amis dans le voisinage et le quotidien. Arrivé au Mali en 1988, ce mouvement fondamentaliste n’a cessé depuis de prospérer sur le terreau du mécontentement social. La Da’wa est peu visible, mais très efficace dans les sociabilités de proximité. Ses adeptes utilisent toutes les mosquées comme leur propre maison (ils y mangent, y dorment) et ont comme tactique la persuasion. Les femmes, qui n’ont pas le droit, elles, de dormir et de manger dans les mosquées, se regroupent autour de leur tahin, terme arabe dont la traduction littérale est la « farine ». Le mouvement a adopté la langue arabe pour qualifier ses repères fondamentaux : le maqamya, de l'arabe maqam, le « lieu », est l'élément de base de l'organisation. Ce maqamya existe dès qu'un comité islamique rattaché à l'idéologie de la Da'wa fonctionne, c'est-à-dire est en capacité d'animer localement des réunions. Ainsi, la Da'wa investit et arabise progressivement les espaces de sociabilité du quotidien à partir desquels elle distille son message. La Da'wa est concurrente du wahhabisme dont elle n'hésite pas à critiquer les modes d'action et de territorialisation.

Les wahhabites portent, eux, un islam de visibilité qui accompagne la diffusion de marquages spatiaux très repérables à travers des mosquées à l'architecture stéréotypée, sans correspondance avec l'architecture locale. Ces dernières servent de repérage signalétique et sont situées préférentiellement, soit à proximité de grands axes, soit en situation d'espaces de conquête : franges urbaines, nouveaux quartiers (Ouallet Anne, 2015). C'est un islam de marquage conquérant, « macdonaldisé », qui s’est développé depuis les années 1950 avec le retour de jeunes intellectuels maliens formés en Arabie saoudite, pays dont ils ont importé la doctrine. D’autres encore ont été formés en Égypte et sont sensibles aux arguments des Frères musulmans. De quelque bord qu'il soit, le fondamentalisme s'est progressivement imposé comme un islam réformateur moderne. Il lamine les mémoires locales (sociales, politiques, artistiques...) qui se recomposent sur des prêt à penser et prêt à porter mondialisés, avant-garde de la prise de contrôle des espaces par l'islam radical. Cet islam mondialisé se branche sur des réseaux transnationaux dont la dimension économique est très puissante.

Au bout du compte, sociétés et territoires sont sous contrôle.

L'islamisation croissante de la société se remarque à travers l'infiltration du religieux dans des domaines auparavant protégés par la laïcité, ce qui était le cas du Mali, conçu au départ comme une république laïque.

\section{Invasion du religieux face à un État central faible et prise de contrôle au nom de l'islam}

La diffusion du hanbalisme agit par perfusion dans les territoires et se traduit aussi, parfois, directement de manière violente dans un djihad les armes à la main. Il s'exprime à travers des

\footnotetext{
${ }^{13}$ Son élan a été ralenti par la concurrence d’une organisation homonyme, Ansar Dine, qui a pris le devant de la scène au moment de l'invasion djihadiste de 2013.
} 
mouvements quiétistes ou djihadistes, le salafisme quiétiste étant en général l'antichambre du salafisme djihadiste.

Le renforcement du pouvoir des associations islamiques se fait par leur implication croissante dans la fourniture des services sociaux, largement abandonnés par les services publics depuis l'application des politiques d'ajustement structurel (PAS) dans les années 1980-1990. Ces PAS ont détruit des secteurs publics comme ceux de l'éducation ou encore de la santé, secteurs déjà fragiles. Face à l'incapacité d'un État ayant finalement délaissé ses responsabilités d'offre des services de base, les associations ont pris le relais, engrangeant une forte légitimité populaire et de très nombreux entrepreneurs, qui se présentent aussi comme des guides spirituels, se sont réappropriés le discours sur le développement, la citoyenneté et la gouvernance. Dans un contexte où les opérations de développement menées par « l'Occident » ont échoué, des fonds arrivent désormais par le biais de nouveaux investisseurs qui utilisent le prétexte de la religion comme arme pour contrôler espaces et populations. Le hanbalisme apparaît comme le support d'un islam radical qui sert de cheval de Troie au libéralisme sauvage. Tous ceux qui le soutiennent surfent clairement sur le vide laissé par le retrait de l’État.

Au Mali, une bonne partie du territoire, à commencer par le Nord, fonctionne finalement comme une zone franche où le contrôle de l'État est totalement absent. Ce sont des espaces de déréglementation livrés au libre jeu d'investisseurs, d'ONG, d'associations diverses qui œuvrent, pour bon nombre d'entre elles, sous couvert de l'islam. Elles se présentent au bout du compte comme la seule force de proposition légitime. Parallèlement, l'islam proposé a la capacité à brancher sur de nouveaux réseaux économiques, dont ceux liés à des activités illégales davantage rémunératrices.

Dans ce cadre du délitement des États et de leur incapacité à contrôler leurs frontières, le terrain est libre pour les trafics en tout genre et les mafieux y trouvent leur compte. Cela fait déjà plusieurs décennies que les passeurs-contrebandiers se jouaient de ces limites étatiques fixées il y a un peu plus d'un demi-siècle et finalement jamais respectées. Le contournement des rares postes de contrôle a toujours été une stratégie des commerçants depuis leur édification. Les frontières ont été transgressées depuis leur création (Grégoire, 2018). Les territoires sont devenus des champs d'expérimentation dans lesquels sont manipulées des revendications à base ethnique (d'abord touareg ${ }^{14}$ au Nord du Mali) et sont investis par des mouvements islamistes divers, parmi lesquels les djihadistes se sont taillés une place de premier plan. Ces derniers, par le projet qu'ils portent, nient totalement les frontières d'États qu'ils ne reconnaissent d'ailleurs pas. Ils ont pour ambition de créer d'autres références spatiales qui s'intègrent parfaitement dans un système de prédation débridée où l'exploitation des rentes de situation est décuplée par l'effacement des frontières. Ils ont pour ambition de prendre le contrôle de tous ces espaces au nom de l'islam.

Parallèlement à des conflits plus généralement exprimés auparavant sur le plan ethnique, un développement des revendications sur le mode religieux a été opéré depuis le début des années 2000 débouchant finalement sur la prise de contrôle de territoires au nom de l'islam.

La revendication de l'Azawad par une partie des Touaregs et des Arabes ${ }^{15}$ du Mali a débouché en 2012 sur une proclamation unilatérale d'indépendance de l'Azawad ${ }^{16}$ le 6 avril 2012. Elle a directement été doublée par une proclamation de la charia en mai $2012^{17}$ et une poussée des djihadistes vers le sud. Un contrôle total des populations par toute une série d'interdictions a alors été déployé dans toute la zone occupée.

Au Mali, c’est donc en 2012 que la collusion a été opérée entre le Mouvement National de

\footnotetext{
${ }^{14}$ Les rébellions touarègues maliennes sont récurrentes depuis l'indépendance du pays : 1963, de 1990 à 1996 , de 2006 à 2008. Celle qui débute en 2012 amène à la proclamation unilatérale d'un État autoproclamé, celui de l'Azawad le 6 avril 2012.

${ }^{15}$ Le Front National de Libération de l'Azawad (FNLA) exclusivement arabe entre dans Tombouctou le 25 avril 2012 , puis en est chassé deux jours après sous la menace d'AQMI.

${ }^{16}$ Ce projet s'appuyait sur le projet d’OCRS mis en place par la France pendant la colonisation (Grégoire, 2018).

${ }^{17}$ Début mai 2012, c'est un chef d’AQMI, Abou Yaya Hamamen, qui est devenu commandant militaire de la ville de Tombouctou. Kidal tombe aussi aux mains d'AQMI ce même mois.
} 
Libération de l'Azawad (MNLA), créé par des Touaregs à Kidal en octobre 2011, et l'islam politique salafiste (MUJAO ${ }^{18}$ et Ansar Dine ${ }^{19}$ ). Le référent ethnique, celui de la revendication touarègue, est alors dépassé pour s’affirmer finalement au nom de l’islam avec AQMI (Al Qaïda au Maghreb Islamique) dans le cadre territorial dénommé « Maghreb islamique ». Du quiétisme salafiste porté par la Da'wa et du marquage wahhabite conquérant on est passé, à partir de 2012, à un djihadisme violent symbolisé par l'application d'une charia dans sa version la plus intransigeante validant les exécutions par lapidation, pendaison, amputation de la main pour les voleurs, égorgements.

C'est la prise de la petite ville de Konna en janvier 2013 qui a accéléré les réactions du Président malien. Les deux tiers du Mali sont alors occupés par les djihadistes et la capitale Bamako risque de tomber entre leurs mains en 24 heures. Le Président malien demande le soutien de la France (10 janvier 2013) qui déclenche l'Opération SERVAL ${ }^{20}$. Une bonne partie du territoire conquis par les djihadistes est repris, les villes de Gao et de Tombouctou sont libérées fin janvier 2013. Mais audelà, le Nord-Est du pays reste toujours sous occupation. La ville de Kidal (symbole du MNLA) et la région de l'Adrar des Ifoghas n’ont jamais été libérées. Plus significatif de la capacité du projet islamique politique porté par le salafisme à s'étendre, les djihadistes ont, depuis, réussi à prendre pied dans la zone «libérée ». Ils se sont implantés dans le centre du Mali avec instauration de la Katiba Macina. La contagion djihadiste vers le centre Mali et, de là par des offensives plus ponctuelles plus au Sud, est confortée par la présence sur place déjà effective de cellules djihadistes. Elle est soutenue par des prédicateurs mêlant souvent causes identitaires autour de l'ethnie et projet salafiste (cas des peuls autour d'Amadou Koufa pour la Katiba Macina). Le Delta intérieur du Niger est ainsi devenu en 2018 un quartier général djihadiste. Le double attentat, le 29 juin 2018, contre le QG du G5 Sahel à Sévaré au centre du Mali et le $1^{\text {er }}$ juillet contre le quartier général de la force Barkhane à Gao le prouve. Il a été, symboliquement, perpétré au moment de la réunion de l'Union Africaine (UA) à Nouakchott ( $1^{\text {er }}$ et 2 juillet 2018).

Le terrain a été préparé depuis au moins une décennie par les salafistes quiétistes et les cellules djihadistes disséminées un peu partout dans le centre et Nord Mali et assez généralement dans l'espace saharo-sahélien. La non contagion de la déstabilisation générale au Niger est due à différents facteurs (Grégoire, 2018) dont les très forts intérêts de la France pour l'uranium à travers Areva. La résistance du Sénégal tient certainement à la force exceptionnelle qu'y revêt le phénomène confrérique et à un État davantage présent. L’Algérie a, elle, été secouée par une attaque djihadiste ${ }^{21}$ très violente avec la prise par les islamistes le 16 janvier 2013 d'In Amenas (site d'extraction du gaz), finalement libéré par l'armée algérienne. Les djihadistes ont alors reconfiguré leurs actions plus au Sud.

L'internationale djihadiste a des bases du Delta intérieur du Niger au Mali jusqu'au Proche-Orient en passant par l'Adrar des Ifoghas et la Libye. Cette concrétisation du projet djihadiste est la négation effective des frontières issues des découpages coloniaux et légitimées par les présidents africains lors de la conférence de l'OUA (actuelle UA) tenue à l'époque à Addis-Abeba en 1963. L'affaiblissement ou l'effondrement des États a permis une mobilité sans précédent des combattants djihadistes de la Libye à la Syrie et dans l'espace saharo-sahélien. Ces mercenaires de tous bords sont rémunérés par ceux qui contrôlent les voies de circulation et donc les espaces. Ils le font à travers des katibas autoproclamées dont les ressources proviennent des différents trafics (otages, drogues, armes, esclaves...).

\section{La territorialisation djihadiste : mobilité et base territoriale}

La nébuleuse djihadiste se défait et se recombine en permanence lorsqu'on l'observe sur les deux dernières décennies. La chute du colonel Kadhafi en octobre 2011 a projeté toute la zone dans

\footnotetext{
${ }^{18}$ Mouvement pour l'Unicité et le Jihad en Afrique de l'Ouest. Le MUJAO chasse le MNLA de Gao le 27 juin 2012.

${ }^{19}$ Antenne locale de l'islam radical présente dans la région dès 2012 et dirigée par Iyad Ag Ghaly, chef touareg.

${ }^{20}$ puis opération Barkhane depuis août 2014 : 3000 hommes déployés dans les 5 pays du G5. Barkhane est basée à N'Djamena avec des moyens aériens à Niamey, à N'Djamena et une base importante installée à Gao (Nord-Est du Mali).

${ }^{21}$ Revendiquée par Mokhtar Belmokhtar.
} 
l'instabilité, a donné un nouveau souffle aux djihadistes et conforté des trafics illicites déjà existants (dont les trafics de drogues et d'armes). L’éclatement de la Libye a de fait détruit les principes présidant aux assises territoriales existantes et remis en jeu le contrôle sur les espaces avant contrôlés par Kadhafi. Parallèlement, les « hivers » islamistes arabes ont conforté l'argument de la légitimation par l'islam politique. La mouvance djihadiste s'est recomposée et les différents groupes sont rentrés dans une compétition beaucoup plus visible.

La labilité des appartenances selon les recompositions des groupes djihadistes ne doit cependant pas occulter le fait que l'objectif est resté le même : fonder un État islamique, un khalifat, et contrôler ressources et populations. Le projet du GSPC (Groupe Salafiste pour la Prédication et le Combat), héritier du GIA algérien, était déjà de transformer l'Algérie en un État islamique. L’évolution de sa concrétisation spatiale a suivi la reprise en main par l'armée algérienne et les alliances passées avec d'autres djihadistes. En 2006, l'allégeance de Droukdal, leader du GSPC, à Ben Laden (Al Qaïda) ancre Al Qaïda en Afrique. Le GSPC se recompose en AQMI un an plus tard et, compte tenu de ses déboires militaires au Nord, revoie sa stratégie en l’orientant vers le Sud. Le Sahara et le Sahel deviennent alors son champ privilégié d'investissement spatial.

La stratégie de DAESH ${ }^{22}$ est aussi de s'implanter sur le continent africain. La création du MCCI (Majilis Choura Chabab al-Islam) par les djihadistes libyens de retour de Syrie en avril 2014 et l'allégeance de cette organisation à Abu Bakr al Baghdadi (DAESH) en novembre de la même année le montrent. L’organisation «État islamique » s'est réellement enracinée en Afrique à ce moment.

Du côté d’Al Qaïda, plusieurs katiba sont créées, correspondant à des camps de combattants extrêmement mobiles, contrôlant les routes de trafics et donc des espaces, dirigés par un chef et inféodés à AQMI, la branche africaine de l'organisation. Ces katiba acquièrent une certaine autonomie d'action et prospèrent à partir d'une base spatiale propre. Cette base territoriale fait fi des frontières des États, s'adapte au contexte par la mobilité et est reliée à l'espace monde des trafics.

Adam Thiam, fin observateur du Mali, le décrit de cette façon : « au Sahara et au Sahel, le modèle islamiste « crée à la place des États - tous les États sur sa route - un empire narco-salafiste qui relie le Sahel des faibles pouvoirs publics aux cartels innovants de la coke latino-américaine » (Thiam Adam, 2013). »

Les groupes islamistes rivalisent ou pactisent les uns avec les autres, les djihadistes rompant ou renouvelant au besoin les allégeances. La dernière reconfiguration est une alliance. En 2017, les principales katiba sahélo-sahariennes décident de faire allégeance à Al Qaida sous une même bannière, celle du Groupe de Soutien à l'Islam et aux Musulmans (GSIM). Plus au Sud, Boko Haram, bastion djihadiste particulièrement actif issu du Nigeria, a prêté allégeance à DAESH. La lutte entre les deux frères ennemis du djihadisme que sont Al Qaïda et DAESH pourrait retarder la possibilité d'établir un khalifat au Sahara-Sahel.

\section{Conclusion}

Les territorialisations de l'islam sont complexes. Elles intègrent ou nient les différents héritages, notamment ceux issus des encadrements politiques, religieux et sociaux d'une histoire longue qui donnent à l'Islam toute sa diversité. Certaines reformulent la mémoire pour mieux investir les espaces dans un contexte de compétition pour l'accès aux ressources. L'islam lui-même est devenu ressource pour nombre de prédicateurs. Certains imposent de nouvelles références pour mieux asseoir leur projet de domination. A l'échelle sous continentale, le contrôle des routes de commerce et donc des marchandises qui y passent est primordial à l'exécution de ce projet. La prise de pouvoir ne se fait pas par le territoire, mais par le contrôle des réseaux.

Dans l'espace Méditerranée-Sahara-Sahel, le branchement sur de tels réseaux implique indéniablement l'adhésion à l'islam, un islam constamment renouvelé, d'abord soufi, confrérique, puis présenté comme réformateur avec des courants salafistes porteur du néolibéralisme (Lavergne Marc, 2009). Cet islam qui a des capacités structurantes en terme d'encadrement des mouvements, des populations et d'exploitation des ressources, n'a que faire des frontières. Il porte un message

\footnotetext{
${ }^{22}$ Acronyme arabe de État Islamique en Irak et au Levant (EIIL).
} 
supranational d'appartenance à une umma, totalement mondialisée dans un islam radical. Dans le contexte néolibéral dominant, cet islam est instrumentalisé par des projets politiques qui dépassent totalement le fait religieux et s'appuie sur une idéologie puisant dans le salafisme ses arguments. Cet islam politique porte des coups de boutoirs aux États-nations modernes. Il s'appuie sur des territorialités mouvantes, dynamiques, qui peuvent être éphémères et se recomposent selon les opportunités.

\section{Bibliographie :}

- Bava Sophie, Boissevain Katia (eds.) (2014), Routes migratoires africaines et dynamiques religieuses CNRS éditions, L'Année du Maghreb, $\mathrm{n}^{\circ} 11$, Dossier en ligne.

- Bourgeot André (2011), Sahara de tous les enjeux, Hérodote, 142, p. $42-77$.

- Bourgeot André, Grégoire Emmanuel (2011), Désordres, pouvoirs et recompositions territoriales au Sahara. Hérodote, 142, p. 3-12.

- Fofana Ibrahim Kader (2017), L'Afrique de l'ouest face à la menace djihadiste, regard prospectif à échéance 2020, L'Harmattan.

- Gallais Jean (1984), Hommes du Sahel, Flammarion.

- Grégoire Emmanuel (2018), Frontières étatiques, frontières mobiles et rentes frontalières dans l'espace saharo-sahélien, L'Information géographique, 1 (mars), pp. 82-98.

- Gueye Cheikh (2002), Touba. La capitale des Mourides, Paris, Karthala.

- Lavergne Marc (2009), Darfour : une guerre villes-campagnes ? Revue Géographique de l'Est vol. 49 / 4, Migrations et réfugiés au Proche-Orient et au Soudan.

- Magrin Géraud (2010), Rentes, territoires et développement : « que tout change pour que rien ne change ", in Pourtier R. (coord.), 1960-2010 : 50 ans d'indépendances africaines, in BAGF, p. 61.

- Ouallet Anne, Al Karjousli Soufian (2018), Territoires du politique et du religieux au Soudan français à travers "Vie et enseignement de Tierno Bokar, le Sage de Bandiagara » d'Amadou Hampaté Bâ, in Jisa Simona, Mișcoiu Sergiu (dir.), Littérature et politique en Afrique, Approche transdisciplinaire, Paris, éd. Cerf, pp. 265-277.

- Ouallet Anne, Al Karjousli Soufian, Cissouma Diama (2015), Diversité, conflictualités et sociabilités au cœur de la patrimonialisation de l'islam au Mali, in Brunet-Jailly Joseph, Charmes Jacques, Konaté Doulaye (dir.), Le Mali contemporain, éd. Tombouctou, IRD, pp. 284-305.

- Ouallet Anne (2013), Les patrimoines : entre pluralité, ressource et lien social, exemples maliens, Chérif Khaznadar (dir.), Le patrimoine, oui, mais quel patrimoine ?, Actes Sud, coll. « Babel », pp. 441-459.

- Retaillé Denis (2018), Sahel, sahel. L’Information Géographique, 1, mars, p. 32-79.

- Retaillé Denis, Walther Olivier, 2013, L’actualité sahélo-saharienne au Mali : une invitation à penser l'espace mobile. Annales de Géographie, 694, p. 595-618.

- Robinson David, Triaud Jean-Louis (éds) (2000), La Tijâniyya, une confrérie musulmane à la conquête de l'Afrique, Karthala, 512 p.

- Scheele Judith (2012), Smugglers and Saints of the Sahara, Regional Connective the Twentieth Century, African Studies, Cambridge University Press.

- Thiam Adam (2013), L'empire narco-salafiste en marche au Sahel, Le Républicain, quotidien malien, janvier 2013.

- Timera Mamadou Bouna, Niang Diene Aminata, Sakho Papa et Diadhiou Diodio (2016), Les territorialités religieuses dans les villes sénégalaises : une étude exploratoire sur l'islam et la production urbaine, Revue de Géographie de l’Université de Ouagadougou, n 5-octobre 2016, Vol. 1, 19 p. 


\section{Carte 1}
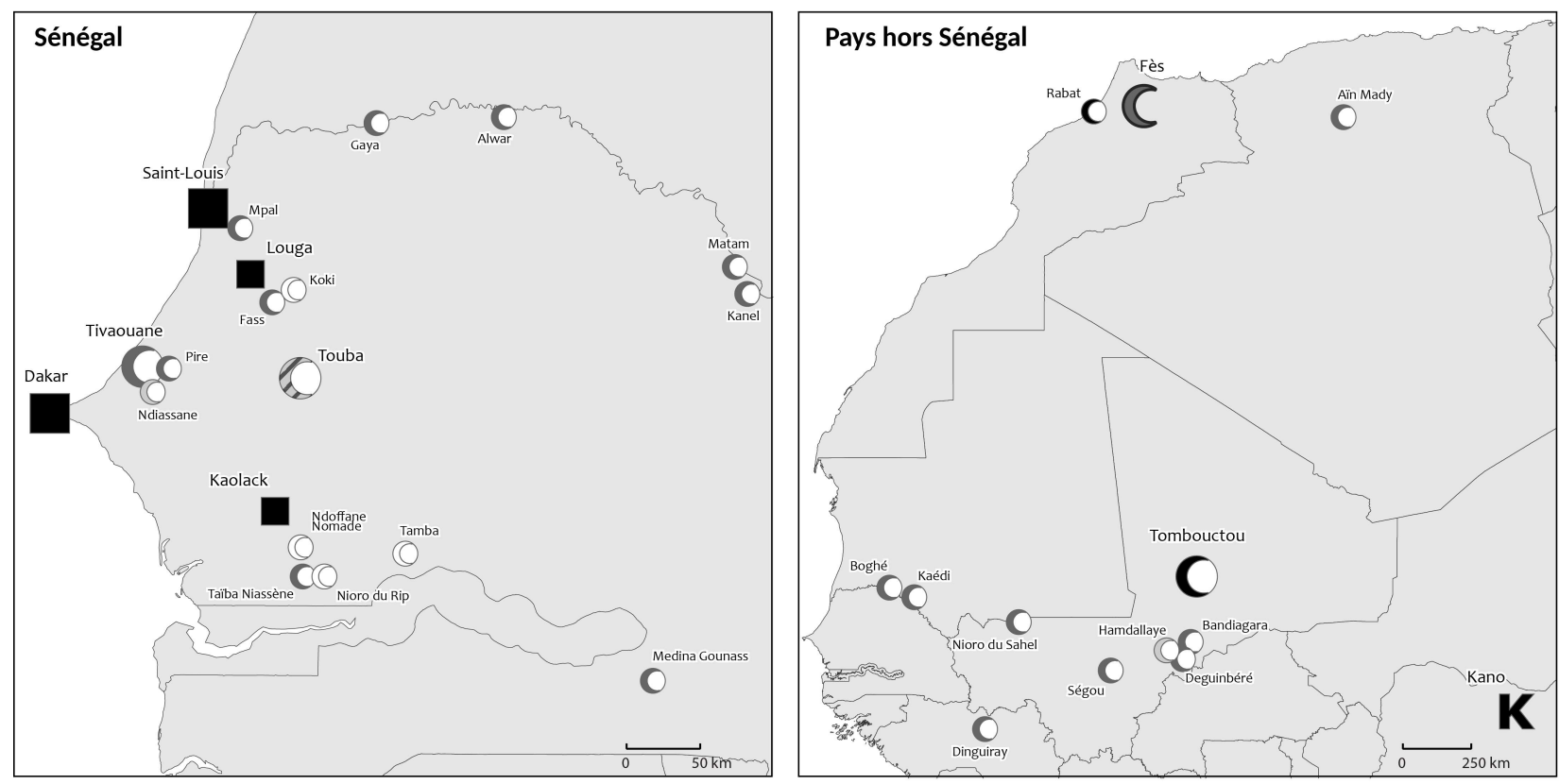

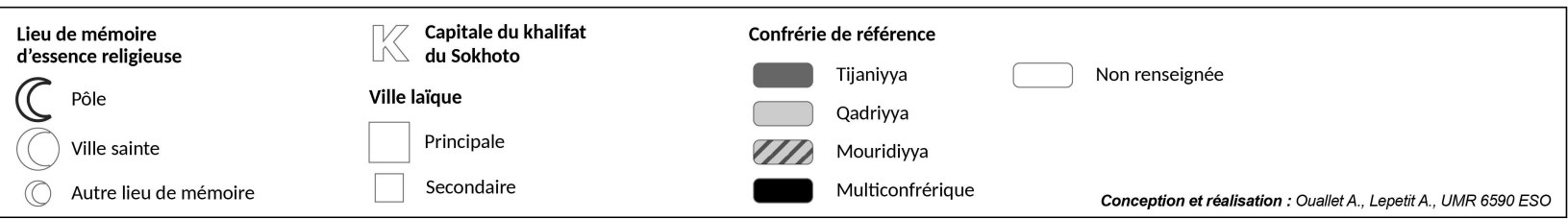

\section{Carte 2}
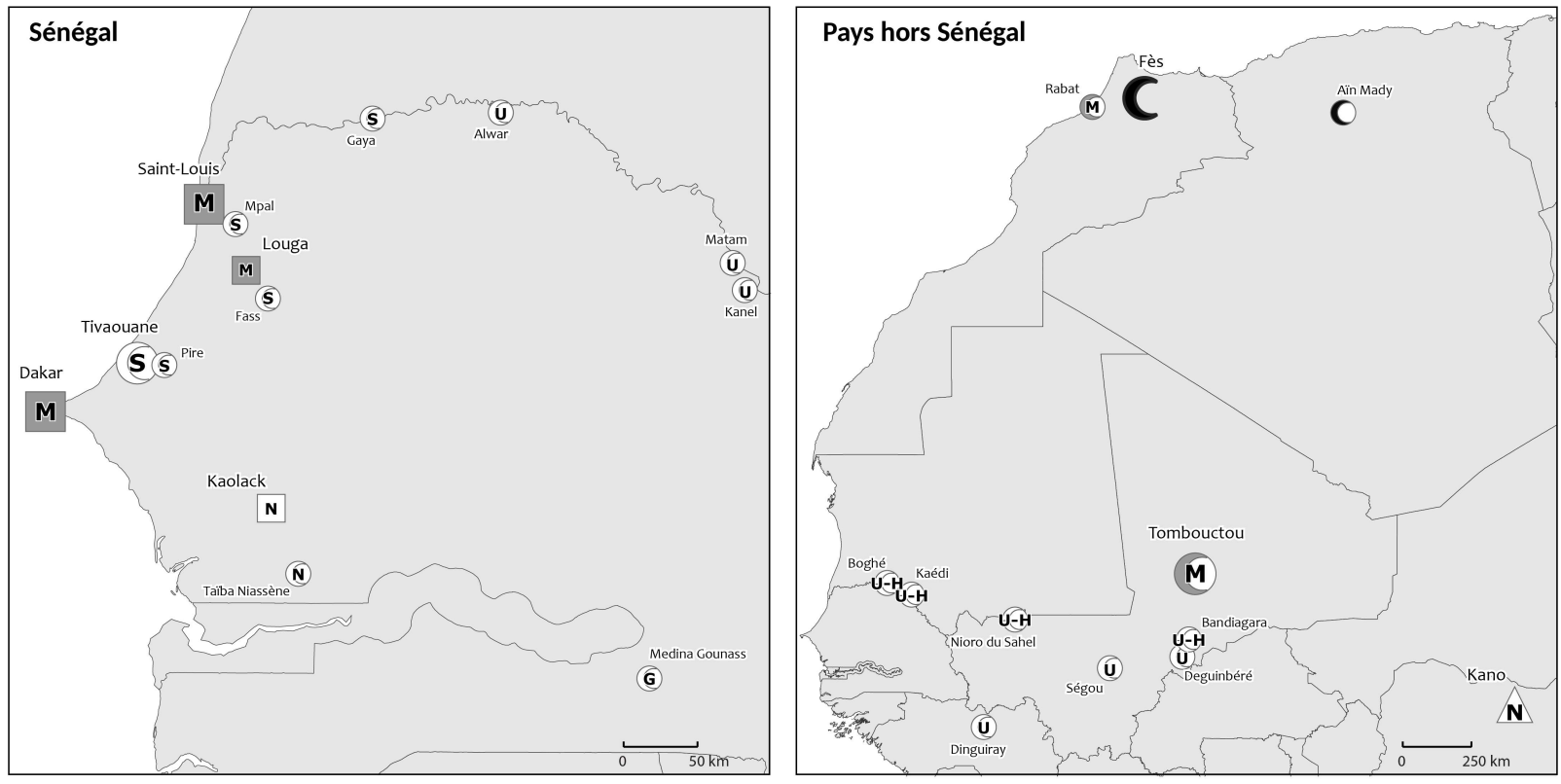

$\begin{array}{ll}\begin{array}{l}\text { Lieu de mémoire } \\ \text { d'essence religieuse }\end{array} & \begin{array}{l}\text { Capitale du khalifat } \\ \text { du Sokhoto }\end{array} \\ \text { Pôle } & \text { Ville laïque } \\ \square \text { Ville sainte } & \text { Principale } \\ \square \text { Autre lieu de mémoire } & \text { Secondaire }\end{array}$

Branche dominante de la Tijaniyya

Maison mère

G Medina Gounass

S Sy

Sy

M Multi-branche 